\title{
Development and Changes with Age of Detrusor Overactivity in Spontaneous Hypertensive Rats as Observed by Simultaneous Registrations of Intravesical and Intraabdominal Pressures
}

\author{
Long-Hu Jin, Hun-Jae Lee ${ }^{1}$, Hwa-Yoan Shin, Bo-Hwa Choi², Sang-Min Yoon, Chang-Shin Park2, Tack Lee \\ Departments of Urology, ${ }^{1}$ Preventive Medicine and ${ }^{2}$ Pharmacology, Inha University College of Medicine by BK 21 Project, Incheon, Korea
}

\begin{abstract}
Purpose: Overactive bladder is especially common in the elderly, although it is not regarded as a normal part of aging. Thus, we investigated how aging alters the cystometric and detrusor overactivity (DO) parameters and the density of nerve growth factor (NGF) in awake spontaneous hypertensive rats (SHRs) of different ages.

Methods: Three age groups of 12- $(\mathrm{n}=5), 17-(\mathrm{n}=6)$, and 21- $(\mathrm{n}=6)$ week-old SHRs (Oriental Bio Inc.) were used. A catheter was implanted into the bladder to record the intravesical pressure (IVP), and a balloon-fitted catheter was positioned in the abdominal cavity to record the intraabdominal pressure (IAP). Of the IVP elevations above $2 \mathrm{~cm} \mathrm{H}_{2} \mathrm{O}$, DO was defined as a rise in IVP without a simultaneous change in IAP and was counted during the filling phase. We measured the expression of NGF in the bladders by enzyme-linked immunosorbent assay.

Results: Both the body and bladder weights significantly increased with age, but the normalized ratio between those was not changed. As for DO, none of the12-week-old rats showed DO, whereas the other groups did. DO increased significantly with age $(\mathrm{P}=0.0045$ by Mantel-Haenszel trend test), although no significant differences were found in DO frequency or pressure between the 17- and 21-week-old age groups. NGF did not show any significant differences among the three groups.

Conclusions: Our results showed that SHRs begin to shows DO after a certain age, such as 12 weeks of age, and that the occurrence of DO has a close relationship with aging. However, NGF, which is known to be increased in the bladder wall of patients with overactive bladder, did not show any relationship with aging in this study.
\end{abstract}

Keywords: Overactive urinary bladder; Urodynamics; Inbred SHR rats; Aging

\section{INTRODUCTION}

Overactive bladder is a urological condition defined by urgency as a core symptom with or without the other symptoms related to voiding [1]. This urgency is known to correspond to the urodynamic events of detrusor overactivity (DO), which is the observation of involuntary bladder contractions during bladder filling [2]. Previous reports estimated that overactive bladder affects about one in six adults, and the prevalence increases with age, although DO is not regarded to be a normal part of aging $[3,4]$. Furthermore, it is anticipated that overactive bladder will become more prevalent in years to come owing to an increasing aging population.

The treatment goal for overactive bladder is to control the debilitating symptoms in order to improve the patient's quality of life, which is more difficult and complex in aging people [5]. Anticholinergic agents are still the primary treatment of choice; these agents reduce the overcontractility of the detrusor muscle
Corresponding author: Tack Lee

Department of Urology, Inha University College of Medicine, Sinheung-dong 3-ga, Jung-gu, Incheon 400-711, Korea

Tel: +82-32-890-3448 / Fax: +82-32-890-3097 / E-mail: 1t11@inha.ac.kr

Co-corresponding author: Chang-Shin Park

Department of Pharmacology, Inha University College of Medicine, Sinheungdong 3-ga, Jung-gu, Incheon 400-711, Korea

Tel: +82-32-890-0962 / Fax: +82-32-887-7488 / E-mail: parkshin@inha.ac.kr

Submitted: November 30, 2011 / Accepted after revision: December 19, 2011
This is an Open Access article distributed under the terms of the Creative Commons Attribution Non-Commercial License (http://creativecommons.org/licenses/by-nc/3.0/) which permits unrestricted non-commercial use, distribution, and reproduction in any medium, provided the original work is properly cited.

Copyright (C 2011 Korean Continence Society 
by targeting the muscarinic receptors in the bladder [6]. However, the use of antimuscarinic drugs is limited by adverse effects such as dry mouth and constipation. Furthermore, age-related changes superimposed on the changes caused by overactive bladder in the lower urinary tract may make the treatment more complex [2]. Thus, we should clarify the effects of the aging process on overactive bladder more clearly, and appropriate animal models are an essential adjunct in trying to address the considerable clinical challenges of this human condition. Spontaneous hypertensive rats (SHRs) are considered a useful tool for studying the pathogenesis of overactive bladder [7] and, furthermore, are a suitable animal model for the study of overactive bladder with aging because of being a genetic model. The hyperactive voiding in SHRs is known to be associated with elevated bladder nerve growth factor (NGF) secretion and related neuroplasticity $[8,9]$.

In the present study, we investigated when DO develops and how aging alters the cystometric and DO parameters in awake SHRs of 12, 17, and 21 weeks of age. We also observed the density of NGF in their bladders.

\section{MATERIALS AND METHODS}

\section{Animals}

Eighteen female SHRs were obtained at the age of 8 weeks from Orient Bio Inc. (Seongnam, Korea). They were reared in our laboratory under controlled conditions with 12 hours of light and 12 hours of darkness and were allowed free access to standard food pellets and tap water. They were subdivided into three agematched categories of 12,17 , and 21 weeks old, resulting in six animals in each age category. All experimental animals and procedures were performed in accordance with the Guide for the Care and Use of Laboratory Animals of the National Institutes of Health and were approved by the local animal ethics committee. One rat of 12 weeks of age died before the cystometry.

\section{Surgical Procedures}

Three days before cystometry, catheter implantations for intravesical pressure (IVP) and intraabdominal pressure (IAP) recording were performed as described previously [10]. The rats were anaesthetized with ketamine $(75 \mathrm{mg} / \mathrm{kg}$ intraperitoneally; Ketamine, Yuhan, Seoul, Korea) and xylazine (15 mg/kg intraperitoneally; Rompun, Bayer Korea Ltd, Seoul, Korea). In brief, the bladder was identified through a lower midline incision. A polyethylene catheter (PE-50; Becton Dickinson, Parsippany,
NJ, USA) with a cuff was inserted into the bladder dome. To record IAP, a balloon (Latex, Dawoo Medical, Incheon, Korea) around the cuff of a catheter tip was placed $1 \mathrm{~cm}$ proximal to the bladder along the catheter to the bladder. The catheters were tunneled subcutaneously and exited through the skin at the back of the animal. The free ends of the catheters were sealed.

\section{In Vivo Cystometric Investigations and Evaluation}

Cystometrograms were obtained in unanesthetized, unrestrained rats in metabolic cages. Before the cystometry, body weight was recorded. The indwelling catheter to the bladder was connected to a two-way valve that was connected via a T-tube to a pressure transducer (Research Grade Blood Pressure Transducer, Harvard Apparatus, Holliston, MA, USA) and a microinjection pump (PHD22/2000 pump, Harvard Apparatus). Another indwelling catheter connected to an air-free abdominal balloon was connected to another pressure transducer to record the IAP. This balloon functions best when it is filled and stabilized to around 50 to $100 \mathrm{~cm} \mathrm{H}_{2} \mathrm{O}$ of IAP. To prevent IAP decay by minute leakages through the multiple joints of the lines and connectors, Parafilm M laboratory sealing film (American National Can, Chicago, IL, USA) was wrapped around the joints. Micturition volumes were recorded by means of a fluid collector connected to a force-displacement transducer (Research Grade Isometric Transducer, Harvard Apparatus). Room-temperature saline was infused into the bladder continuously at a rate of $10 \mathrm{~mL} \cdot \mathrm{h}^{-1}$. IVP, IAP, and micturition volumes were recorded continuously with Acq Knowledge 3.8.1 software and an MP150 data acquisition system (Biopac Systems Inc., Goleta, CA, USA) at a sampling rate of $100 \mathrm{~Hz}$. The mean values from three reproducible micturition cycles were used for evaluation. IAP was defined as the recorded balloon pressure corrected by subtracting the lowest balloon pressure in each voiding cycle, which is comparable to zeroing in human cystometry. Detrusor pressure was defined as IVP minus IAP. The IVP rises during the filling phase were defined as increments of IVP that exceeded $2 \mathrm{~cm} \mathrm{H}_{2} \mathrm{O}$ from baseline, which was interpreted as abdominal straining if occurring with simultaneous similar changes in IAP, or as DO if occurring without simultaneous similar changes in IAP.

The following two kinds of cystometric parameters were investigated.

Pressure- and volume-related parameters: Basal pressure (BP; the lowest bladder pressure during filling), threshold pressure (TP; bladder pressure immediately before micturition), maxi- 
mum pressure (MP; maximum bladder pressure during the micturition cycle), micturition volume (MV; volume of expelled urine), residual volume (RV; remaining urine after voiding), bladder capacity (BC; MV+RV), and micturition interval (MI; intervals between micturition contractions).

DO-related parameters during the filling phase: Time of filling phase (interval from the initiation of infusion through the tube and the point immediately before the initiation of micturition), frequency of abdominal straining per minute, frequency of DO per minute, and increased amplitude from base to peak of DO spike as IVP. These frequencies were calculated on the basis of the time of filling phase. After cystometry, the animals were sacrificed by cervical dislocation. The bladder and urethra were removed en bloc and separated at the level of the bladder neck and the bladder was weighed.

\section{Enzyme-Linked Immunosorbent Assay (ELISA)}

Samples were homogenized with homogenization buffer (150 $\mathrm{mM}$ sodium chloride, $1 \%$ triton $\mathrm{X}-100,1 \%$ sodium deoxycholate, $0.1 \%$ sodium dodecyl sulfate, $50 \mathrm{mM}$ Tris- $\mathrm{HCl}, \mathrm{pH} 7.5$, and ethylenediaminetetraacetic acid), phosphatase inhibitor cocktail (PhosSTOP, Roche, Mannheim, Germany), and protease inhibitor cocktail (Sigma-Aldrich Co., St. Louis, MO, USA). The samples were centrifuged at $10,000 \mathrm{~g}$ for 4 minutes. Protein concentrations were determined by using the Pierce BCA Protein Assay kit (Pierce Chemical Co., Rockford, IL, USA). The samples were stored in a deep freezer at $-80^{\circ} \mathrm{C}$ until assayed. The samples were assayed by Abfrontier's rat NGF ELISA kit (Cat No.LF-EK50293). Rat NGF-specific monoclonal antibodies were precoated onto $96-$-well plates. The rat specific detection polyclonal antibodies were biotinylated. The samples and bioti- nylated detection antibodies were added to the wells subsequently and then followed by washing with phosphate-buffered saline. Avidin-biotin-peroxidase complex was added and unbound conjugates were washed away with phosphate-buffered saline. HRP-substrated TMB was used to visualize HRP enzymatic reaction. TMB was catalyzed by HRP to produce a blue color product that changed into yellow after adding acidic stop solution. Plates were read at $450 \mathrm{nM}$ on an Elx800 microplate reader (Bio-Tek Instruments Inc., Winooski, VT, USA). All tissue NGF values were standardized by tissue protein levels and expressed in $\mathrm{pg} / \mathrm{mg}$ protein.

\section{Statistical Analyses}

The results are expressed as mean values \pm standard errors of the mean. Normal distributions were confirmed by the ShapiroWilks' W test. Statistical analyses were undertaken with unpaired Student's t-tests or a one-way analysis of variance with the Tukey post-hoc test for multiple comparisons. The data for DO incidence were expressed as percentages of animals showing DO. Trends in percentage were tested by using a Mantel-Haenszel chi-squared test. All analyses were performed with GraphPad Prism, version 5.03, 2009 (GraphPad Software Inc., La Jolla, CA, USA). $\mathrm{P}<0.05$ was considered statistically significant.

\section{RESULTS}

\section{Body and Bladder Weights}

The body weight of the SHRs increased with age from 12 to 21 weeks. The bladder weight showed a pattern similar to the results for body weight. When bladder weight was normalized to body weight, however, there were no significant differences among
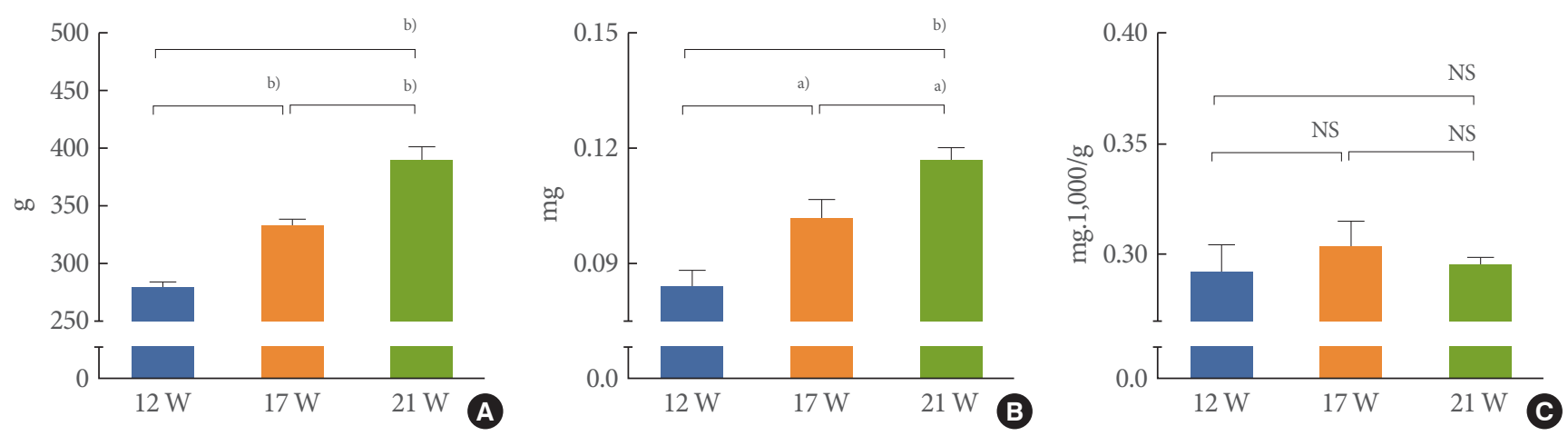

Fig. 1. Effects of age on bladder (A) and body weights (g) (B) and the normalized ratio of bladder weight (mg) to body weight (g) (C) in spontaneous hypertensive rats (SHRs). Vertical bars represent mean results plus or minus standard error of the mean. $12 \mathrm{~W}$, 12-week-old SHRs; 17 W, 17-week-old SHRs; $21 \mathrm{~W}, 21$-week-old SHRs; NS, not significant. ${ }^{\mathrm{a}}{ }^{\mathrm{P}}<0.05 .{ }^{\mathrm{b}} \mathrm{P}<0.01$. 

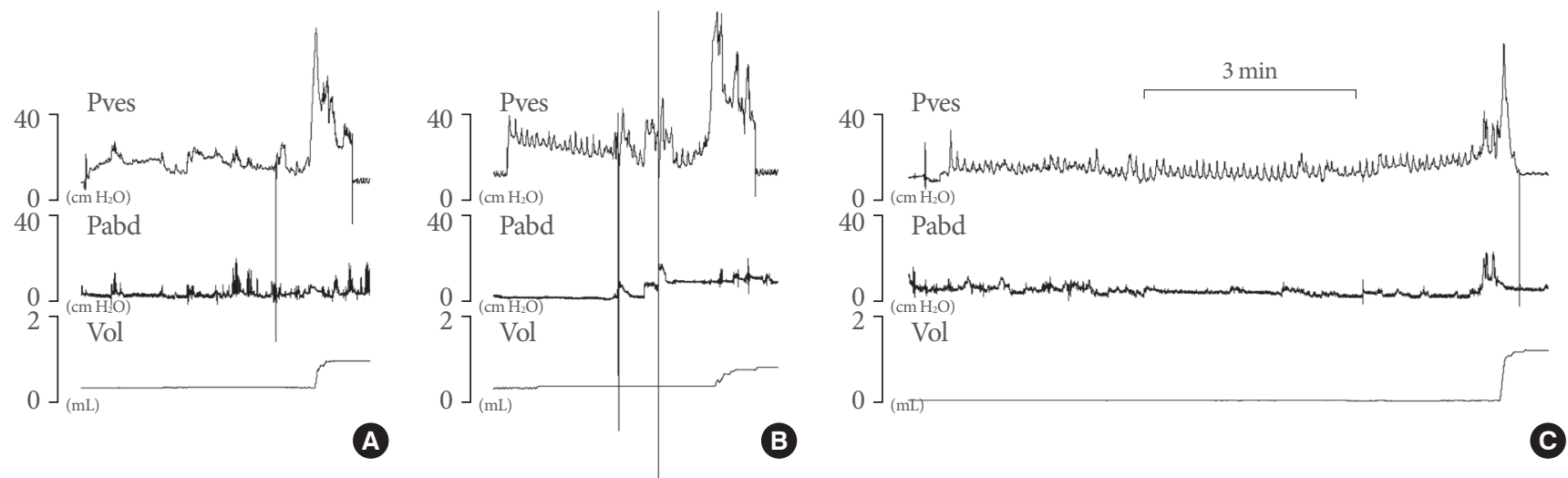

Fig. 2. Representative cystometric tracings in conscious spontaneous hypertensive rats (SHRs). (A) 12-week-old SHRs. (B) 17-weekold SHRs. (C) 21-week-old SHRs. Pves, intravesical pressure; Pabd, intraabdominal pressure; Vol, volume.
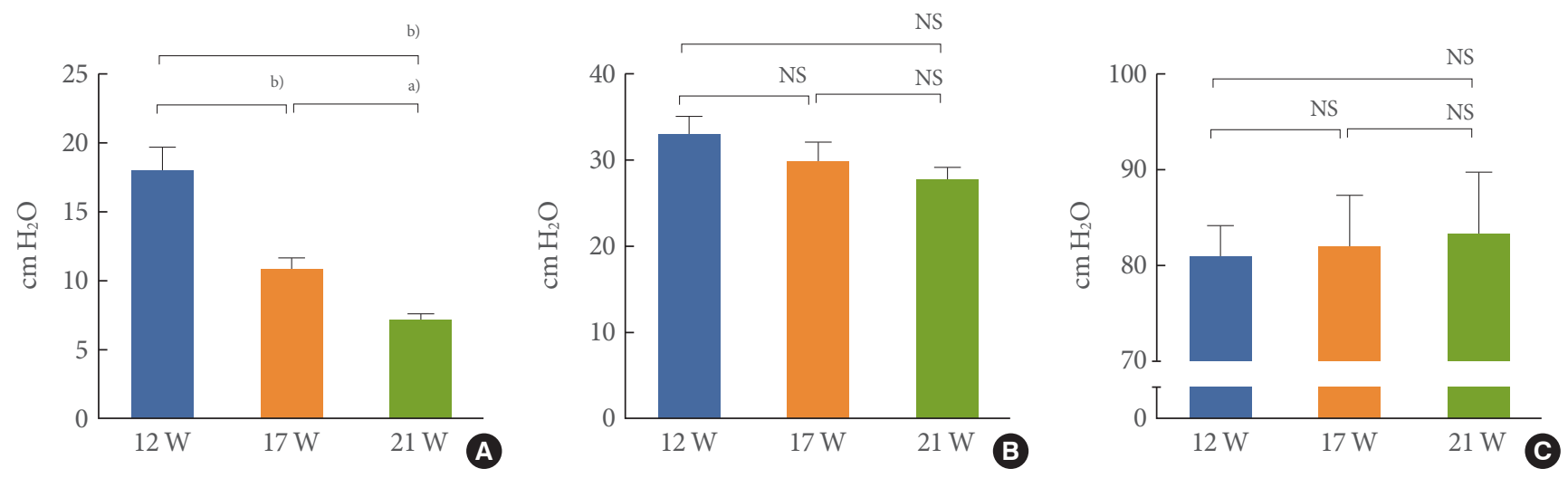

Fig. 3. Effects of age on pressure-related parameters including basal pressure (A), threshold pressure (B), and maximal pressure (C). Bars represent mean results plus or minus standard error of the mean. $12 \mathrm{~W}, 12$-week-old SHRs; 17 W, 17 -week-old SHRs; 21 W, 21-week-old SHRs; NS, not significant. ${ }^{\text {a) }} \mathrm{P}<0.05$. ${ }^{\text {b) }} \mathrm{P}<0.01$.

the three groups of 12-, 17-, and 21-week-old SHRs (Fig. 1).

\section{Cystometric Pressure, Volume, and DO-Related Parameters}

Representative cystometric tracings from SHRs of 12, 17, and 21 weeks of age are shown in Fig. 2. All the curves for micturition cycles with IAP measurements were reproducibly recorded in all animals, and the comparisons of all pressure parameters were performed on the basis of detrusor pressure, which is derived by the differences between IVP and IAP.

The BPs of the SHRs decreased with age from 12 to 21 weeks. The TPs and MPs showed no significant differences among the three age groups (Fig. 3). Volume parameters of SHRs including BC, MV, and MI showed increased values at 21 weeks of age compared with 12 and 17 weeks of age, but no significant differences were observed between the 12- and 17-week-old age groups. There were no RVs in any age group (Fig. 4).

The time of the filling phase showed increased values only in the 21-week-old age group (data not shown), similar to the data for BC, MV, and MI. The frequency of abdominal straining was higher in the 12-week-old age group than in the 17- and 21week-old age groups (Fig. 5A).

DO proved by IAP was not shown in the 12-week-old age group, but was shown in the 17- and 21-week-old age groups. The incidence of DO was higher in rats in the 21-week-old age group ( 5 of 6 rats, $83.3 \%$ ) than in the 17-week-old age group (3 of 6 rats, 50.0\%). Thus, in these female SHRs, DO increased significantly with age $(\mathrm{P}=0.0045$ by Mantel-Haenszel trend test) (Fig. 5B). However, there were no significant differences in DO frequency or pressure between the 17- and 21-week-old age groups (Fig. 5C, D).

\section{NGF Levels in the Three Age Groups of SHRs}

Subsequent evaluation by ELISA of NGF protein in bladder was successful in all rats except two rats in the17-week-old age group. 

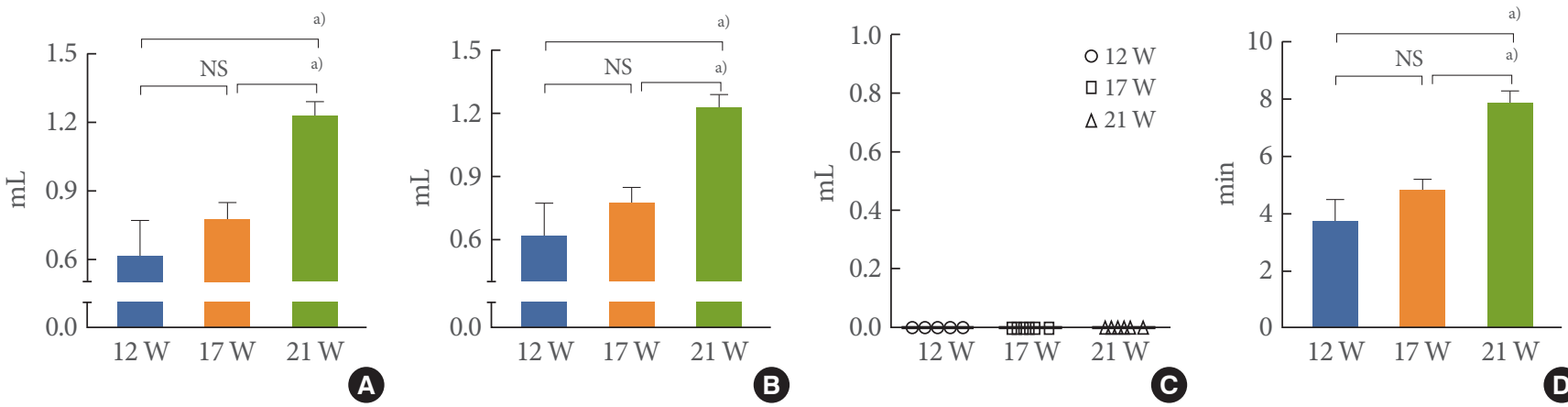

Fig. 4. Effects of age on volume-related parameters including bladder capacity (A), voiding volume (B), residual volume (C), and micturition interval (D). Bars represent mean results plus or minus standard error of the mean. $12 \mathrm{~W}$, 12-week-old SHRs; $17 \mathrm{~W}, 17$-weekold SHRs; $21 \mathrm{~W}, 21$-week-old SHRs; NS, not significant. ${ }^{\text {a) }} \mathrm{P}<0.01$.
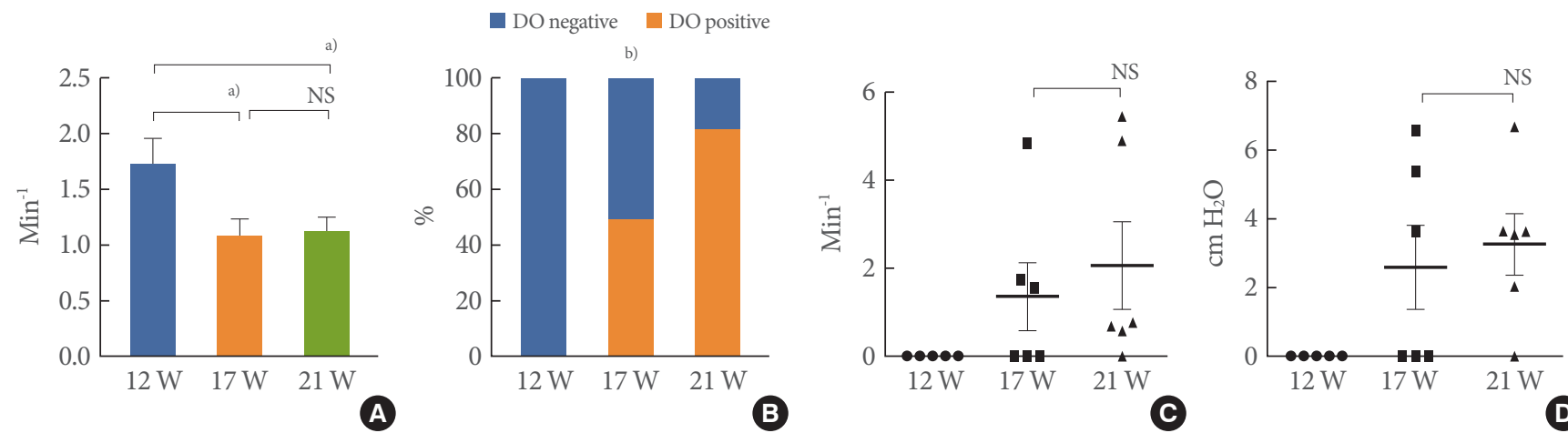

Fig. 5. Effects of age on detrusor overactivity (DO)-related parameters including abdominal straining (A), incidence of DO (B), DO frequency $(\mathrm{C})$, and DO pressure (D). Bars represent mean results plus or minus standard error of the mean. 12 W, 12-week-old SHRs; $17 \mathrm{~W}, 17$-week-old SHRs; $21 \mathrm{~W}, 21$-week-old SHRs; NS, not significant. ${ }^{\mathrm{a})} \mathrm{P}<0.05,{ }^{\mathrm{b}} \mathrm{P}=0.0045$.

There were no significant differences among the three age groups, although the 21-week-old age group $(1.91 \pm 1.35 \mathrm{pg} / \mathrm{mg})$ showed a tendency for increased values compared with the 12-week-old age group $(1.45 \pm 0.21 \mathrm{pg} / \mathrm{mg})$ and the 17 -weekold age group (1.36 $\pm 0.18 \mathrm{pg} / \mathrm{mg}$ ) (Fig. 6).

\section{DISCUSSION}

Our study showed that DO objectively proved by IAP came into existence after 12 and before 17 weeks of age in our strain of SHRs, thus showing a greater prevalence with increasing age. Therefore, this study provides direct proof of the close relationship between overactive bladder and aging in the animal model of SHRs. However, the age at which SHRs begin to show DO may differ in other strains of SHRs, because of the diverse biological characteristics that result from different breeding techniques and environments in different laboratories. In contrast with our findings for DO, NGF, which is known to be increased

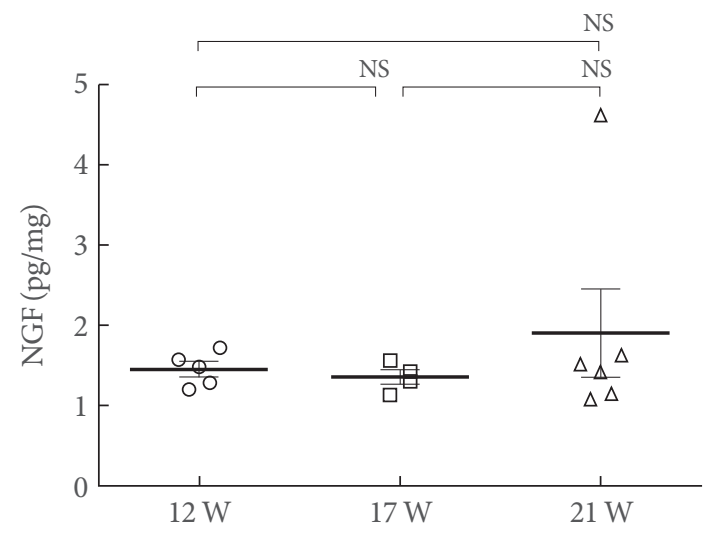

Fig. 6. Effects of age on nerve growth (pg/mg) in the bladders of spontaneous hypertensive rats. Bars represent mean results plus or minus standard error of the mean. $12 \mathrm{~W}, 12$-week-old SHRs; 17 W, 17-week-old SHRs; 21 W, 21-week-old SHRs; NS, not significant; NGF, nerve growth factor.

in the bladders of rats with overactive bladder [8], did not show any relationship with aging in this study. 
The SHR model is currently regarded as a valuable animal model for exploring the pathogenesis of overactive bladder $[7,9]$. The rats were originally developed as a genetic model of hypertension from Wistar rats and their Wistar-Kyoto inbred non-hypertensive controls [10]. In the course of establishing inbred strains of this rat model, some substrains showed a variety of characteristics such as the incidence of stroke, hyperactive behaviors, impulsivity, and hyperactive voiding $[11,12]$, which have been selected as relevant rat models to mimic various human diseases, such as stroke, attention deficiency hyperactive disorder (ADHD), and overactive bladder. However, although most of the information related to these features could be gathered from adult SHRs, it is important to verify the age at which those features start to be replicated and the changes that occur afterwards in SHRs. Hypertension is known to develop at about 4 to 10 weeks of age without any artificial intervention [13]. SHRs are also used as an ADHD model, because the hyperactive behavior comes into existence at about 3 to 4 weeks of age before the hypertensive condition [14]. However, no studies have been carried out on which age of the rat is appropriate to use as a model of overactive bladder. Our results showed that DO was shown in the SHRs from the age of 17 weeks. This suggests that DO develops during the adolescent period, because 6 months of age in the rat corresponds to 18 years of human age [15]. After that, the rats showed an increasing incidence of DO with age, which is applicable to the correlations of aging with the incidence of overactive bladder in humans. We suggest that the overactive bladder research in this substrain of SHRs should be done from at least 12 weeks of age.

Many research reports on the bladder have used awake cystometry rather than anesthetic cystometry as a research tool, because the bladder is controlled by the complex physiology of central nervous system superimposed on the autonomic nervous system [16,17]. In cystometry, the elevations of IVP, which is measured through a catheter in the bladder, can reflect detrusor contractions or abdominal straining. If no discriminations between those by IAP are performed for alterations in IVP, abdominal straining by innate hyperactive behaviors could limit the usefulness of SHRs as a DO model. We reported previously that confirmed DO by our method for IAP measurement represented up to $76 \%$ of the pressure elevations, among total IVP elevations more than $2 \mathrm{~cm} \mathrm{H}_{2} \mathrm{O}$ during the filling phase, whereas the remaining $24 \%$ were caused by abdominal straining in SHRs [10]. Our results are based on this accurate methodology for DO. Furthermore, the observed abdominal straining in our animals showed a decrease with aging, which suggests that the rats become less active with age.

Our study showed that from 12 to 21 weeks of age, the bladders of SHRs exhibited serial changes in bladder and body weights and pressure and volume parameters. The bladder and body weights of the SHRs increased from 12 to 21 weeks of age, but when bladder weight was normalized to body weight, there were no significant differences among the three groups of 12-, 17-, and 21-week-old SHRs. This suggests that the bladder grows isometrically with the body. BP, which represents the basal tension of the bladder, decreased with aging, accompanied by increased BC. In contrast, the incidence of DO during the filling phase increased with age, which is direct evidence that overactive bladder increases with age. DO can have myogenic or neurogenic etiologies, which include detrusor muscle instability and damage to neuronal pathways $[18,19]$. In SHRs, overproduction of NGF in bladder tissue from SHRs was demonstrated and the intriguing possibility has been suggested that this leads to hyperinnervation of NGF-dependent sympathetic or sensory nerves in the bladder, resulting in hyperactive voiding in SHRs [8]. However, we did not observe increasing NGF in our models with age, whereas the incidence of DO did increase. The detailed pathophysiological mechanisms related to NGF in the aging bladder should be elucidated further in future studies.

In conclusion, our results suggest that the DO in SHRs begins to occur after a certain age, such as 12 weeks of age in our study. That age may be different in other strains of SHRs, because of the diverse biological characteristics that result from different breeding techniques in different laboratories. However, we should remember this point when planning to use SHRs as a DO animal model. Because of the close relationship with aging, SHRs will be a good model that will enhance our understanding of the relationship between overactive bladder and aging.

\section{CONFLICT OF INTEREST}

No potential conflict of interest relevant to this article was reported.

\section{ACKNOWLEDGEMENTS}

This work was supported by an Inha University Research Grant. 


\section{REFERENCES}

1. Abrams P, Cardozo L, Fall M, Griffiths D, Rosier P, Ulmsten U, et al. The standardisation of terminology in lower urinary tract function: report from the standardisation sub-committee of the International Continence Society. Urology 2003;61:37-49.

2. Semins MJ, Chancellor MB. Diagnosis and management of patients with overactive bladder syndrome and abnormal detrusor activity. Nat Clin Pract Urol 2004;1:78-84.

3. Stewart S, Hart CL, Hole DJ, McMurray JJ. Population prevalence, incidence, and predictors of atrial fibrillation in the Renfrew/Paisley study. Heart 2001;86:516-21.

4. Tubaro A. Defining overactive bladder: epidemiology and burden of disease. Urology 2004;64(6 Suppl 1):2-6.

5. Shah D, Badlani G. Treatment of overactive bladder and incontinence in the elderly. Rev Urol 2002;4 Suppl 4:S38-43.

6. Thüroff JW, Abrams P, Andersson KE, Artibani W, Chapple CR, Drake MJ, et al. EAU guidelines on urinary incontinence. Eur Urol 2011;59:387-400.

7. Steers WD. Pathophysiology of overactive bladder and urge urinary incontinence. Rev Urol 2002;4 Suppl 4:S7-18.

8. Clemow DB, Steers WD, McCarty R, Tuttle JB. Altered regulation of bladder nerve growth factor and neurally mediated hyperactive voiding. Am J Physiol 1998;275(4 Pt 2):R1279-86.

9. Steers WD, Clemow DB, Persson K, Sherer TB, Andersson KE, Tuttle JB. The spontaneously hypertensive rat: insight into the pathogenesis of irritative symptoms in benign prostatic hyperplasia and young anxious males. Exp Physiol 1999;84:137-47.

10. Jin LH, Andersson KE, Kwon YH, Park CS, Yoon SM, Lee T. Substantial detrusor overactivity in conscious spontaneously hypertensive rats with hyperactive behaviour. Scand J Urol Nephrol 2009;43: 3-7.

11. Okamoto K, Aoki K. Development of a strain of spontaneously hypertensive rats. Jpn Circ J 1963;27:282-93.

12. Yamori Y. Implication of hypertensive rat models for primordial nutritional prevention of cardiovascular diseases. Clin Exp Pharmacol Physiol 1999;26:568-72.

13. Zicha J, Kunes J. Ontogenetic aspects of hypertension development: analysis in the rat. Physiol Rev 1999;79:1227-82.

14. Adriani W, Caprioli A, Granstrem O, Carli M, Laviola G. The spontaneously hypertensive-rat as an animal model of ADHD: evidence for impulsive and non-impulsive subpopulations. Neurosci Biobehav Rev 2003;27:639-51.

15. Quinn R. Comparing rat's to human's age: how old is my rat in people years? Nutrition 2005;21:775-7.

16. Andersson KE. Advances in the pharmacological control of the bladder. Exp Physiol 1999;84:195-213.

17. de Groat WC, Yoshimura N. Pharmacology of the lower urinary tract. Annu Rev Pharmacol Toxicol 2001;41:691-721.

18. Brading AF. A myogenic basis for the overactive bladder. Urology 1997;50(6A Suppl):57-67.

19. de Groat WC. A neurologic basis for the overactive bladder. Urology 1997;50(6A Suppl):36-52. 